\title{
A novel chimeric antigen receptor (CAR) system using an exogenous protease, in which activation of $T$ cells is controlled by expression patterns of cell-surface proteins on target cells
}

\author{
SATORU AOYAMA ${ }^{1,2}$, SHUNICHIRO YASUDA ${ }^{1,2}$, HUIXIN LI ${ }^{1}$, DAISUKE WATANABE ${ }^{1,2}$, \\ YOSHIHIRO UMEZAWA ${ }^{2}$, KEIGO OKADA ${ }^{2}$, AYAKO NOGAMI $^{2,3}$, OSAMU MIURA $^{2}$ and NORIHIKO KAWAMATA ${ }^{1,2}$ \\ Departments of ${ }^{1}$ Immunotherapy for Hematopoietic Disorders and ${ }^{2}$ Hematology, and ${ }^{3}$ Laboratory Medicine, \\ Tokyo Medical and Dental University (TMDU), Tokyo 113-8510, Japan
}

Received November 5, 2021; Accepted January 17, 2022

DOI: 10.3892/ijmm.2022.5097

\begin{abstract}
Anti-CD19 chimeric antigen receptor (CAR)-T cell therapy against refractory B-cell malignancies shows excellent therapeutic effects. However, there are some obstacles to be overcome in this treatment. Since current CAR-T cells target a single cell-surface protein on tumor cells, the CAR-T cells also attack normal cells expressing the protein. This is one of the major adverse effects of this therapy. To improve target-cell-specificity of this therapy, we established a novelCAR system, in which T-cell activation was controlled by expression patterns of proteins on target cells. Our novel CAR-T cells had two distinct CARs consisting of a 'Signal-CAR', recognizing a protein on tumor cells, and a 'Scissors-CAR', recognizing another protein on normal cells. The signal-CAR had a peptide sequence which was cleaved by the Scissors-CAR, and functional domains for cellular activation. The Scissors-CAR had a protease domain that cleaved its recognition peptide sequence in the Signal-CAR. When tumor cells expressed only the protein recognized by the Signal-CAR, the tumor cells were attacked. By contrast, normal cells expressing both the proteins induced inactivation of the Signal-CAR through cleavage of the recognition site when getting in contact with the CAR-T cells. To establish this system, we invented a Scissors-CAR that was dominantly localized on cell membranes and was activated only when the CAR-T cells were in contact with the normal cells. Using a T-cell line, Jurkat, and two proteins, CD19 and HER2, as target proteins, we showed that the anti-CD19-Signal-CAR was cleaved by the anti-HER2-Scissors-CAR when the CAR-T cells were co-cultivated with cells expressing both the
\end{abstract}

Correspondence to: Professor Norihiko Kawamata, Department of Immunotherapy for Hematopoietic Disorders, Tokyo Medical and Dental University (TMDU), 1-5-45 Yushima, Bunkyo-ku, Tokyo 113-8510, Japan

E-mail: nkawamata@gmail.com

Key words: chimeric antigen receptor, HIV protease, human primary $\mathrm{T}$ cell, multi-antigen recognition, cancer immunotherapy proteins, CD19 and HER2. Furthermore, we demonstrated that primary CAR-T cells expressing both the CARs showed attenuated cytotoxicity againsT cells with both the target proteins. Our novel system would improve safety of the CAR-T cell therapy, leading to expansion of treatable diseases by this immunotherapy.

\section{Introduction}

Chimeric antigen receptor (CAR)-T cell therapies are efficient methodologies for the immunotherapy of refractory malignancies. Anti-CD19-CAR-T cell therapy shows excellent therapeutic effects against B-cell lymphoblastic leukemia $(1,2)$ and B-cell lymphoma $(3,4)$. CAR-T cells recognizing B-cell maturation antigen (BCMA) are used for refractory multiple myeloma and efficiently eradicate myeloma cells (5). Current CARs have a domain that recognizes a molecule on tumor cells, a transmembrane domain, a domain of a co-stimulation molecule such as $\mathrm{CD} 28$ or $4-1 \mathrm{BB}$, and a cytoplasmic domain of $\mathrm{CD} 3-\zeta(6)$.

Since current CARs recognize a single target molecule on tumor cells, the CAR-T cells also attack normal cells expressing the antigen. For example, normal B cells in patients who are treated with anti-CD19-CAR-T cells are attacked since the cells express CD19. To protect patients from severe infections, those patients need life-long supplemental immunoglobulin infusions, which is an anti-infectious product of normal B cells (7). This is called an 'on-target/off-tumor effect'. This adverse effect is one of the major obstacles to be overcome in this treatment. Improvement of target-cell-specificity of CAR-T cell therapy could diminish this 'on-target/off-tumor effect', leading to less toxicity of this therapy. Here, we show a novel regulatory CAR-T cell system with higher target-cell-specificity by recognizing the expression patterns of two distinct proteins on target cells.

\section{Materials and methods}

Cell lines. A human T-cell acute lymphoblastic leukemia cell line, Jurkat, a human Burkitt's lymphoma cell line, Raji, and a human chronic myelogenous leukemia cell line, K562 were 
purchased from American Type Culture Collection (ATCC) and maintained in RPMI-1640 media with $10 \%$ fetal bovine serum (FBS). The $293 \mathrm{~T}$ cell line and a human breast cancer cell line, SK-BR-3 were purchased from ATCC and maintained in DMEM supplemented with $10 \%$ FBS. All of the cells were maintained at $37^{\circ} \mathrm{C}$ with $5 \% \mathrm{CO}_{2}$.

Reagents and antibodies. Recombinant human interleukin (IL)-2 was purchased from R\&D Systems. For manipulation of human primary T cells, the Dynabeads Untouched Human T cell kit and Human T-Activator CD3/CD28 were purchased from Thermo Fisher Scientific, Inc. For western blot analysis, anti-RFP/mCherry rabbit polyclonal antibody (PM005; 1:1,000 dilution) was purchased from Medical and Biological Laboratories; anti-GFP/YFP rabbit polyclonal antibody (SC-8334; 1:5,000 dilution) and anti-syntaxin 4 mouse $\mathrm{mAb}$ (QQ-17; 1:1,000 dilution) were purchased from Santa Cruz Biotechnology, Inc.; anti- $\beta$-actin mouse mAb (A1978; 1:10,000 dilution) was purchased from Sigma Aldrich; Merck KGaA; anti-rabbit horseradish peroxidase (HRP)-conjugated antibody (NA934; 1:5,000 dilution) and anti-mouse HRP conjugated antibody (NA9310; 1:5,000 dilution) were purchased from GE Healthcare. For flow cytometric analysis, PE anti-human CD69 antibody (FN50; 1:20 dilution), PerCP/Cy5.5 anti-human HER2 antibody (24D2; 1/50 dilution), and FITC anti-human CD19 antibody (4G7; 1/50 dilution) were purchased from BioLegend, Inc. Hoechst 33258 was purchased from Dojindo Molecular Technologies, Inc. HIV protease (HIVPR) inhibitors, saquinavir and nelfinavir, were kindly provided by Professor Yamaoka at the Department of Virology, TMDU (Tokyo, Japan).

Preparation and expansion of human primary $T$ cells. Ten milliliters of peripheral blood collected from healthy donors was diluted with $10 \mathrm{ml}$ of phosphate-buffered saline (PBS), and $10 \mathrm{ml}$ of the diluted blood was applied onto $3 \mathrm{ml}$ of Lymphoprep (Abbott Diagnostics Technologies AS). After centrifugation at $800 \times \mathrm{g}$ (at room temperature) for $20 \mathrm{~min}$, white blood cells were collected. Human primary $\mathrm{T}$ cells were purified using Dynabeads Untouched Human T cell kit (Thermo Fisher Scientific, Inc.) according to the manufacturer's protocols. CAR-T cells were established as previously reported $(8,9)$. Briefly, $1.0 \times 10^{5}$ cells $/ \mathrm{ml}$ of T cells were expanded in RPMI-1640 media with 10\% FBS, $30 \mathrm{U} / \mathrm{ml}$ IL-2, and CD3/CD28 beads (1:3 cell:bead ratio) for 3 days. Expanded $\mathrm{T}$ cells were transduced with lentivirus. In the present study, we collected peripheral blood from three healthy donors. Donor 1 was a 35-year-old male. His peripheral blood was collected in November, 2020 and June, 2021. Donor 2 was a 36-year-old male. His peripheral blood was collected in December, 2020 and April, 2021. Donor 3 was a 25-year-old-female. Her peripheral blood was collected in January and August, 2021. All the procedures including peripheral blood collection, T-cell isolation and manipulation were performed at TMDU, after informed consents were obtained. All the procedures involving human cells were approved by the TMDU Ethics Committee (M2019-294).

Vector construction. Regions encoding $\mathrm{scFv}$ domains were amplified from plasmids, pHR_PGK_antiCD19_ synNotch_TetRVP64 (Addgene plasmid \#79126),
pHR_PGK_antiHer24D5-3_synNotch_Gal4VP64 (Addgene plasmid \#85422), and pHR_PGK_antiHer24D5-8_synNotch_ Gal4VP64 (Addgene plasmid \#85425). The plasmids were gifted by Dr W.A. Lim (10). All the scFv regions were subcloned and ligated into other plasmids. A plasmid encoding CD28, pcDNA3.1-PS11-scFvFc-CD28-gp41(706-713) (Addgene plasmid \#60606) and a plasmid encoding HIVPR, pcDNA3/GFP-PR (Addgene plasmid \#20253) were gifted by Dr W.A. Marasco (11) and Dr N.P. Dantuma (12), respectively. To establish constructs of regulatory CARs, sequences of the scFv fragments, the CD28 transmembrane and co-stimulatory domains, the HIVPR, and the fluorescence proteins were connected using PCR. A HIVPR recognition peptide sequence (SFSFPQIT) was inserted as a cleavage site in the constructs. A CD3- $\zeta$ fragment was amplified from pGEM-human TCR zeta/2470 (Addgene plasmid \#11507) gifted by Dr A.M. Weissman (13) and inserted in the constructs. A gag/pol vector, psPAX2 (Addgene plasmid \#12260), and a VZV envelope vector, pMD2.G (Addgene plasmid \#12259), for lentivirus packaging were gifted by Dr D. Trono. Schematic descriptions of the constructs are shown in Fig. S1.

Transfection and lentiviral infection. 293T cells were transfected using Lipofectamine 3000 reagent (Thermo Fisher Scientific, Inc.) according to the manufacturer's protocols. Twenty-four hours after transfection, the cells were examined under a microscopy and/or harvested for western blot analysis. To obtain SK-BR-3 cells with CD19, pcDNA3-CD19-Hygro, was transfected into the cells using Lipofectamine 3000 and selected in medium containing $100 \mu \mathrm{g} / \mathrm{ml}$ of hygromycin. To obtain Raji cells with HER2, pcDNA3-HER2-Hygro was transduced using Nucleofector kit V (Lonza) according to the manufacturer's protocols and selected in medium containing $300 \mu \mathrm{g} / \mathrm{ml}$ of hygromycin. Jurkat cells with CARs were obtained by transduction of pcDNA3-anti-CD19-Signal-CA R-T2A-YFP using Nucleofector kit V (Lonza) and selected in medium containing $1 \mathrm{mg} / \mathrm{ml}$ of G418.

For lentivirus packaging, vectors encoding CARs, psPAX2, and pMD2.G were co-transfected into 293T cells using Lipofectamine 3000 according to the manufacturer's protocols. Briefly, one day before gene transduction, $2.0 \times 10^{6}$ of 293T cells were plated on $10-\mathrm{cm}$ collagen-coated plates (Iwaki) in $10 \mathrm{ml}$ of DMEM with $10 \%$ FBS. 293T cells were transduced with $10 \mu \mathrm{g}$ of a vector encoding CARs, $7.5 \mu \mathrm{g}$ of psPAX2 and $2.5 \mathrm{mg}$ of pMD2.G. Twenty-four hours after the transfection, supernatants containing lentivirus were collected and $10 \mathrm{ml}$ of fresh medium was added on the cells. Forty-eight hours after the transfection, the second viral supernatants were collected. All the supernatants were filtrated through $0.45-\mu \mathrm{m}$ syringe filters (Pall Life Sciences). Then, the viral particles were precipitated using Lenti-X concentrator (Takara) according to the manufacturer's protocols. For lentivirus infection, $2 \mathrm{ml}$ of 10 -time concentrated lentiviral supernatants were applied onto 6-well plates coated with Retronectin (Takara), and the virus particles were captured on the plates by centrifugation at $1,080 \mathrm{x} \mathrm{g}\left(\right.$ at $32^{\circ} \mathrm{C}$ ) for $120 \mathrm{~min}$. After removal of the supernatant, $2.0 \times 10^{6}$ of Jurkat cells or primary $\mathrm{T}$ cells were seeded onto the plates for infection. One day after the infection, the cells were transferred to new plates for further experiments. 
Western blot analysis. Cells were lysed in a $40 \mu 1$ of lysis buffer containing $1 \%$ Triton $\mathrm{X}-100,20 \mathrm{mM}$ Tris- $\mathrm{HCl}(\mathrm{pH} 7.5)$, $150 \mathrm{mM} \mathrm{NaCl}, 1 \mathrm{mM}$ EDTA, $1 \mathrm{mM}$ sodium orthovanadate, $1 \mathrm{mM}$ phenylmethylsulfonyl fluoride, $10 \mu \mathrm{g} / \mathrm{ml}$ of aprotinin, and $10 \mu \mathrm{g} / \mathrm{ml}$ of leupeptin. Following incubation (at $4^{\circ} \mathrm{C}$ for $15 \mathrm{~min}$ ) and centrifugation at $17,400 \mathrm{x} \mathrm{g}\left(\right.$ at $4^{\circ} \mathrm{C}$ ) for $15 \mathrm{~min}$, supernatants were mixed with equal volume of $2 \mathrm{X}$ Laemmli sample buffer and heated at $100^{\circ} \mathrm{C}$ for $5 \mathrm{~min}$. Separation of membranous and cytosolic fractions from samples was performed using Trident Membrane Protein Extraction kit (Genetex) according to the manufacturer's protocols. Ten microliters of the samples was electrophoresed in 12.5\% PAGE gel (Atto) and transferred onto Immnobilon-P membranes (Merck Millipore). The membranes were blocked with 5\% skim milk at room temperature for $1 \mathrm{~h}$. Primary antibodies were diluted with Tris-base buffered saline with Tween (TBS-T) and incubated at $4^{\circ} \mathrm{C}$ for overnight. Subsequently, the membranes were incubated with diluted secondary antibodies at room temperature for $1 \mathrm{~h}$. The transferred proteins detected by antibodies were visualized by the Western Lightning Plus-ECL chemiluminescence kit (PerkinElmer, Inc.) according to the manufacturer's protocols. All the data shown are representatives of results of at least three independent experiments.

Microscopic examination. Cells $(200,000)$ were seeded on 35-mm glass-bottom plates (Matsunami) in $10 \mathrm{ml}$ of DMEM with $10 \%$ FBS. One day after cultivation, plasmids were transfected into the cells using Lipofectamine 3000 according to the manufacturer's protocols. For nuclear staining, Hoechst 33258 solution was added into the medium at 1:500 dilution. The cells were examined with an all-in-one fluorescence microscopy BZ-X800 (Keyence, Osaka, Japan; magnification, x600). Optical sectioning images (14) were captured and analyzed using a BZ-X800 Analyzer (Keyence). All the data and images shown are representatives of results of at least three independent experiments.

Flow cytometric analysis. For flow cytometric analysis, $4.0 \times 10^{5}$ cells were stained with $50 \mu \mathrm{l}$ of antibody solution diluted with PBS with $3 \% \mathrm{FBS}$ at $4^{\circ} \mathrm{C}$ for $30 \mathrm{~min}$. The cells were washed with $1 \mathrm{ml}$ of the buffer and resuspended in $500 \mu \mathrm{l}$ of the buffer. The cells were analyzed on a FACS Calibur flowcytometer (BD Bioscience). The cells with YFP and/or mCherry were sorted by a FACS Aria II (BD Bioscience). The data were analyzed using FlowJo ver.8.8.7 software (BD Bioscience). All the data shown are representatives of results of at least three independent experiments.

Cytotoxicity assays. To assess the cytotoxicity of CAR-T cells, lactate dehydrogenase (LDH) release assays were carried out using Cytotoxicity LDH Assay kit-WST (Dojindo) according to the manufacturer's protocols. CAR-T and target cells were mixed at indicated effector-to-target (E:T) ratios and co-cultivated. Ten thousand SK-BR-3 cells or $2.0 \times 10^{4}$ Raji or K562 cells were used as target cells in $100 \mu 1$ of medium in 96-well plates. Twenty-four hours after co-cultivation, the supernatants were harvested and levels of LDH released from the target cells were measured using Multiskan FC (Thermo Fisher Scientific, Inc.). Specific cytotoxicity (\%) was calculated as follows: [(Experimental release)-(Effector spontaneous release)-(Target spontaneous release)]/[(Target maximum release)-(Target spontaneous release)] x100. To measure maximum releases from target cells, the target cells were lysed and levels of LDH released from the cells were measured according to the manufacturer's protocols.

Statistical analysis. Differences in the datasets containing two groups were analyzed with the Student's t-test. Differences in the datasets containing more than three groups were analyzed with one-way analysis of variance (ANOVA) with Dunnett's post-hoc test or Tukey post-hoc test. $\mathrm{P}<0.05$ was regarded as indicative of a statistically significant difference. Statistical analyses were performed using EZR software version 1.55 (15).

\section{Results}

Concepts of a novel CAR system recognizing two distincT cell-surface proteins on tumor/normal cells. Current CARs expressed in human $\mathrm{T}$ cells are composed of four elements including i) an extracellular domain recognizing a cell-surface protein on tumor cells, ii) a trans-membrane domain, iii) a cytoplasmic co-stimulatory domain, and iv) a cytoplasmic CD3- $\zeta$. To increase target-cell-specificity of CAR-T cells, we designed two CARs which recognize two distincT cell-surface proteins (Proteins A and B in Fig. 1). T cells expressing these two CARs distinguish target cells depending on expression patterns of these two proteins. To regulate a functional property of CARs, we designed two CARs with two distinct cytoplasmic domains. One is CAR with a protease domain of HIVPR in the cytoplasmic domain (Scissors-CAR); the other has a HIVPR recognition peptide sequence between the co-stimulatory domain and the CD3- $\zeta$ in the cytoplasmic domain (Signal-CAR) as shown in Fig. 1. $\mathrm{T}$ cells expressing this Signal-CAR transduce the T-cell activation signal (Fig. 1A). We hypothesized that $\mathrm{T}$ cells expressing both Signal- and Scissors-CARs were in contact with cells expressing both the proteins $\mathrm{A}$ and $\mathrm{B}$, leading to accumulation of these two types of CARs on the contacting surface of $\mathrm{T}$ cells. Interaction between HIVPR and HIVPR recognition peptide sequence leads protein cleavage of Signal-CAR and CD3- $\zeta$ in Signal-CAR was released and spread in the cytoplasm. Multimer formation of CD3- $\zeta$ under the plasma membrane is a trigger for T-cell activation, thus, this Scissors-CAR-mediated protein cleavage suppresses the CAR-T cell activation (Fig. 1B). To establish this methodology, we designed and constructed vectors as described above, and performed experiments to prove this principle.

Establishment of a novel regulatory CAR construct: Scissors-CAR. We constructed a vector with anti-CD19 scFv (FMC63), CD28, and a protease domain of HIVPR. To examine localization of this CAR, a YFP fluorescence protein was inserted at the C-terminus (Fig. 2A, top) and fluorescence optical sectioning imaging by a microscope was performed, which allowed us to capture sliced images of viable cells (14). This $\alpha$ CD19-CD28-HIVPR-YFP showed cytoplasmic distribution of YFP (Fig. 2B, left panel). Western blot analysis using an anti-YFP antibody detected a band of $40 \mathrm{kDa}$ (Fig. 2C), not an expected size of anti-CD19-CD28-HIVPR-YFP (76 kDa). These results suggested that this CAR was auto-cleaved at 
A

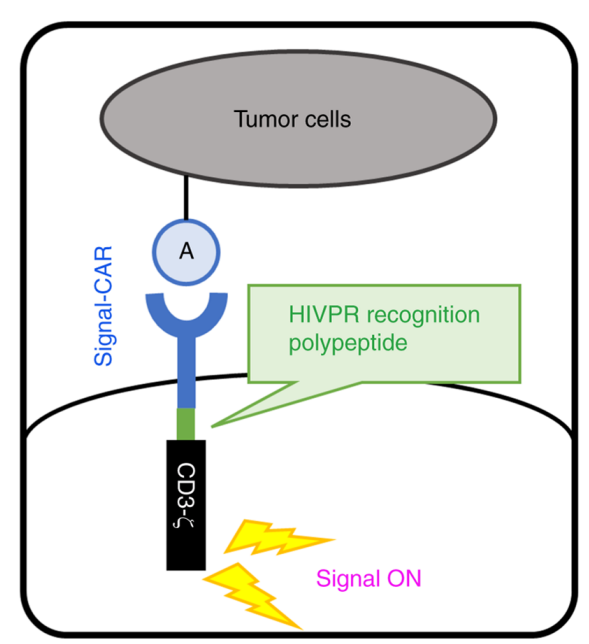

$\mathrm{B}$

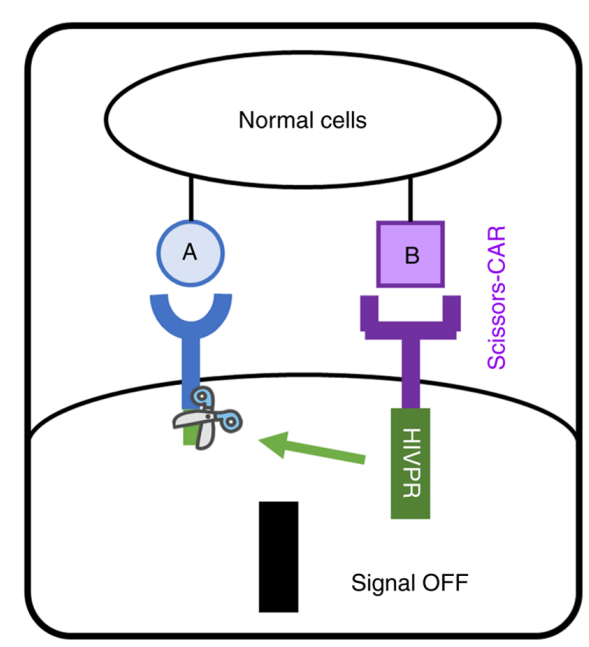

Figure 1. Concepts of a novel CAR-T cell system. (A) Activation of CAR-T cells. A tumor cell (gray oval) expressing only antigen-A (blue circled A) activates a CAR-T cell expressing anti-A-Signal-CAR. Signal-CAR has a scFv domain binding to the antigen-A (blue bident), HIVPR protease recognition peptide sequence (green line) upstream of an activation domain of CD3- $\zeta$ (black box). A curved black line depicts a cell membrane of the CAR-T cell. When the CAR interacts with the antigen-A, stimulatory signals (yellow thunder shapes) are transduced into the cell (signal ON in pink letters). (B) Signal regulation by a protease (HIVPR). When the novel CAR-T cell contacts with a normal cell (white oval) expressing both antigens-A (blue circled A) and -B (purple boxed B), both Signal- and Scissors-CARs (blue and purple bidents) are assembled on the contacting surface of the cell (curved black line), Scissors-CAR cleaves the recognition peptide sequence (green line) in Signal-CAR, leading to inactivation of signaling of Signal-CAR via the protein cleavage (signal OFF in black letters). A green long arrow indicates activation of the protease. CAR, chimeric antigen receptor.

the N-terminal of the HIVPR domain. We speculated that this CAR had cleavage site(s) recognized by HIVPR in its cytoplasmic region. One candidate site was four amino acids sequence (PQIT) in HIVPR $(12,16)$. We deleted this site and examined localization of the CAR (Fig. 2A, middle and B, middle). Deletion of the four amino acids (4AA) in HIVPR enhanced the membranous localization of the CAR (Fig. 2B, middle) and partially suppressed the auto-cleavage (Fig. 2C). Furthermore, we found another candidate site in the CD28 inner cellular domain (ICD) as shown in Fig. 2A that was predicted by ExPASy PeptideCutter tool (https://web.expasy. org/peptide_cutter/) (17). Elimination of both the candidate sequences of CD28 and HIVPR lead to the membranous localization of the CAR (Fig. 2B, right) and strongly suppressed the auto-cleavage (Fig. 2C). We named this construct Scissors-CAR and was used as a regulatory CAR in further experiments.

A protease-domain of HIVPR cleaves the recognition sequence in CAR and induces translocation of the cytoplasmic domain . To examine whether cleavage of CARs leads to translocation of a cytoplasmic domain of CARs, we constructed a CAR with anti-CD19 scFv (FMC63), CD28, and mCherry fluorescence protein in the cytoplasmic domain (Fig. 3A). The HIVPR recognition peptide sequence was inserted upstream of mCherry, and we named this construct anti-CD19-mCherry-CAR (Fig. 3A). This anti-CD19-mCherry-CAR showed membranous localization after transfection into $293 \mathrm{~T}$ cells (Fig. 3B). Since the results shown above suggested that the HIVPR-YFP protein cleaved from anti-CD19-CD28-HIVPR-YFP was localized in the cytoplasm (Fig. 2B), we expected that the HIVPR-YFP in the cytoplasm would cleave the recognition sequence in anti-CD19-mCherry-CAR. As expected, western blot analysis using cytoplasmic and plasma membrane fractions of the transfected cells showed that the mCherry-CAR was cleaved when anti-CD19-CD28-HIVPR-YFP was co-expressed (Fig. 3C). Furthermore, HIVPR inhibitors, saquinavir or nelfinavir, suppressed the cleavage in a dose-dependent manner (Fig. 3D), suggesting that the cleavage was induced by the HIVPR-YFP. Cells transduced both anti-CD19-mCherry-CAR and anti-CD19-CD28-HIVPR-YFP showed cytoplasmic distribution of the mCherry signal (Fig. 3E). These results suggested that the HIVPR-YFP in the cytoplasm cleaved mCherry-CAR and induced translocation of the cytoplasmic domain of the CAR. By contrast, cells expressing both anti-CD19-mCherry-CAR and anti-CD19-Scissors-CAR showed membranous localization of mCherry (Fig. 3F). This result suggested that Scissors-CAR on the membrane was enzymatically inactive without target cells.

Scissors-CAR cleaves anti-CD19-mCherry-CAR, leading to translocation of mCherry when target cells express two distinct proteins. To assess our regulatory CAR system using Scissors-CAR, we used CD19 and HER 2 cell-surface target proteins. We established Raji cells stably expressing HER2 as target cells. The parental Raji cells expressed only CD19, not HER2; the engineered Raji cells expressed both CD19 and HER2 (Fig. 4A). To evaluate target-cell-dependent cleavage of CAR, 293T cells expressing both anti-CD19-mCherry-CAR and anti-HER2-Scissors-CAR were co-cultivated with Raji cells (Fig. 4B). The 293T cells co-cultivated with Raji cells expressing only CD19, the mCherry CAR signal showed membranous localization (Fig. 4C). By contrast, the 293T cells co-cultivated with the engineered Raji cells expressing both CD19 and HER2, the mCherry signal was translocated from the membrane to the cytoplasm (Fig. 4D). Not only microscopic analysis, but also western blot analysis indicated that the 293T cells transduced with anti-CD19-mCherry-CAR and anti-HER2-Scissors-CAR showed mCherry-CAR cleavage, which was induced by engineered Raji cells expressing both 
A

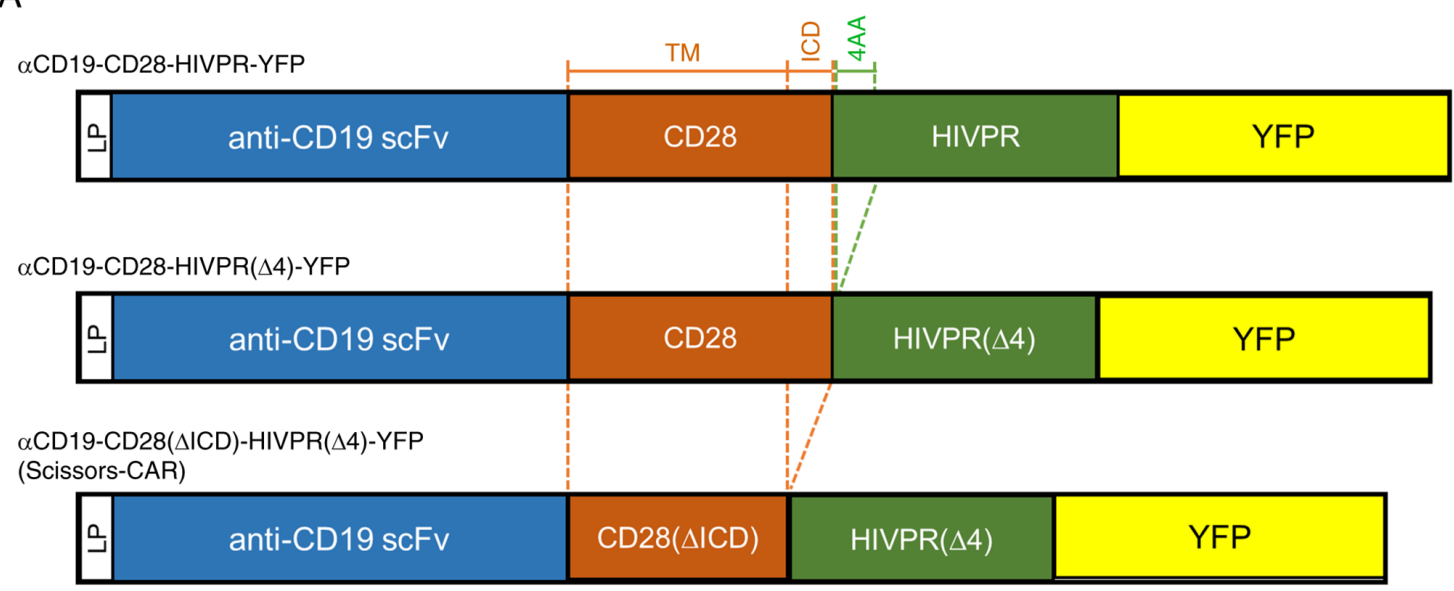

B

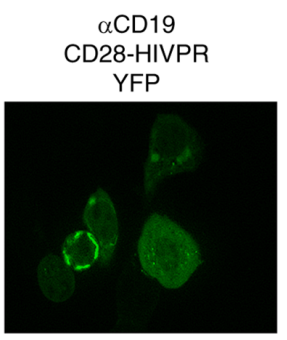

$\alpha \mathrm{CD} 19$ $\operatorname{CD28-HIVPR}(\Delta 4)$ YFP
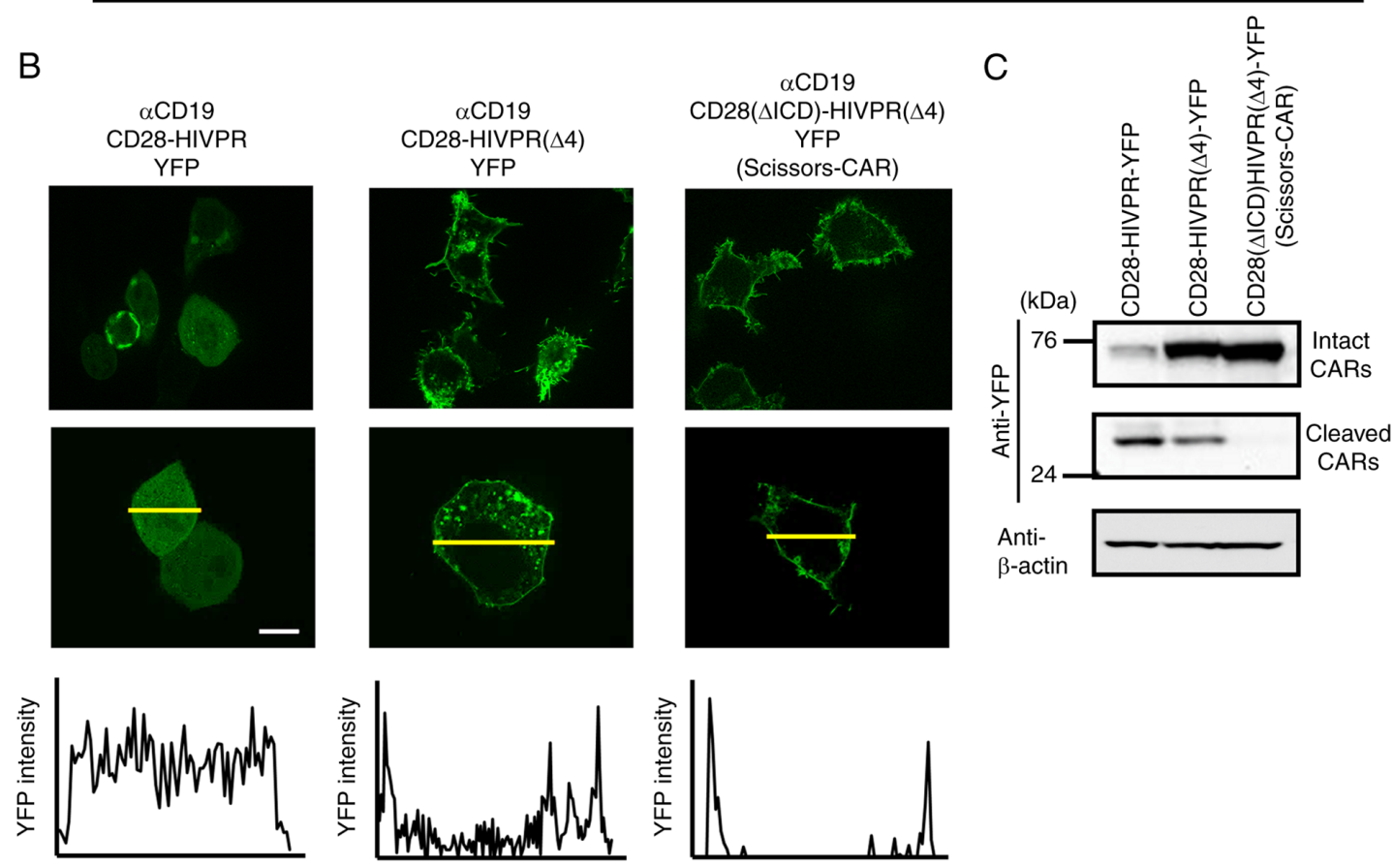

Figure 2. Establishment of a regulatory CAR: Scissors-CAR. (A) Structures of regulatory CARs. All three constructs had a leader peptide (LP) sequence (white boxes), an anti-CD19 scFv (FMC63) domain (blue boxes), a CD28 transmembrane (TM) domain (orange boxes), a HIVPR protease domain (green boxes) and a YFP fluorescence protein (yellow boxes). A top construct has a CD28 inner cellular domain (ICD) and four amino acids (4AA), which were candidate recognition sequences of HIVPR. A construct at the middle is a deletion mutant lacking the 4AA sequence $(\Delta 4)$. A construct at the bottom is a deletion mutant lacking both CD28-ICD $(\triangle \mathrm{ICD})$ and 4AA $(\Delta 4)$. (B) Microscopic examination of 293T cells expressing indicated CAR constructs. Twenty-four hours after gene transduction, localizations of the CARs (green color) were examined by the fluorescence optical sectioning imaging. Intensities of YFP fluorescence on the yellow lines in the middle panels are plotted in the bottom panels. Scale bar, $20 \mu \mathrm{m}$. (C) Western blot analysis of whole cell lysates of $293 \mathrm{~T}$ cells expressing indicated CAR constructs. Twenty-four hours after gene transduction, expressed proteins were detected by an anti-YFP antibody. Intact CARs (76 kDa) and cleaved CARs ( $35 \mathrm{kDa}$ ) were detected. $\beta$-actin was used as a loading control. CAR, chimeric antigen receptor.

CD19 and HER2 (Fig. S2). Furthermore, the cleavage was saturated at $24 \mathrm{~h}$ after initiation of co-cultivation as shown in Figs. 4 and S2. On the other hand, in the absence of target cells, minimal levels of cleaved mCherry-CAR were detected (left four lanes in each panel in Fig. S2).

The data also showed that ligand-independent cleavage of mCherry-CAR was associated with the amounts of transfected Scissors-CAR (left four lanes of each panel in Fig. S2). The experiments showed that higher numbers of target cells increased the cleavage of mCherry-CAR (right four lanes of each panel in Fig. S2). However, the higher numbers of target cells also increased ligand-independent cleavage (left four lanes of the bottom panel in Fig. S2). These results suggested that our CAR system using Scissors-CAR cleaved the other CAR when target cells expressed both the cell-surface target proteins, CD19 and HER2.

Activation of the novel CAR-T cells is controlled depending on patterns of proteins expressed on target cells. To evaluate functional properties of our system, we constructed the anti-CD19-Signal-CAR with anti-CD19 scFv (FMC63), CD28, HIVPR recognition peptide sequence, and $\mathrm{CD} 3-\zeta$. As a marker, a T2A-YFP fluorescence protein cassette was connected in the C-terminus (Fig. 5A). Since T2A site acted as a ribosomal skipping motif (18), this construct expressed both the full-length CAR protein and YFP protein. Therefore, cells 
A

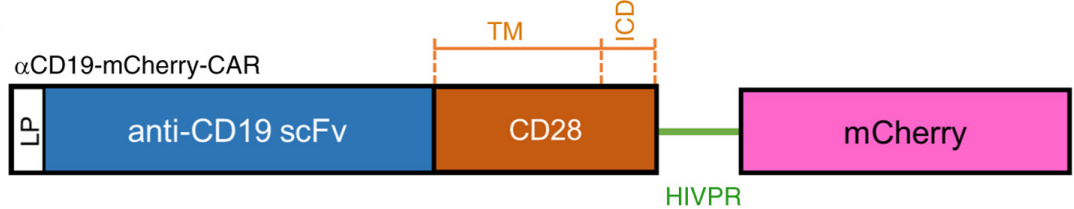

recognition peptide

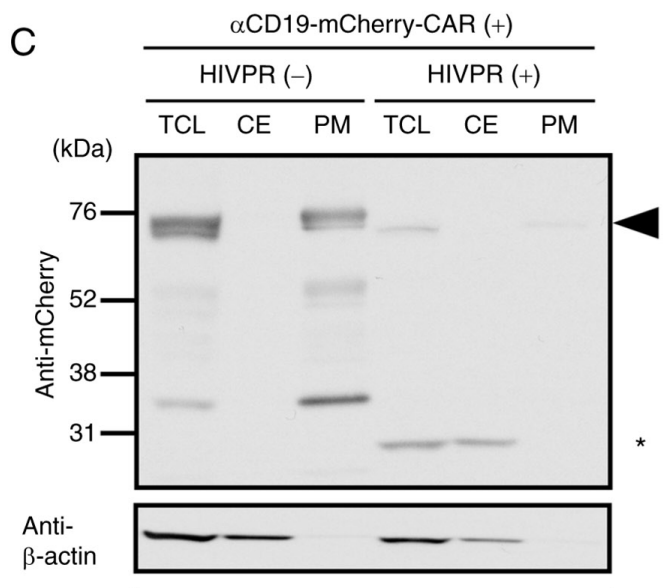

Anti-
Syntaxin4 $\square-\square$

$\mathrm{E}$

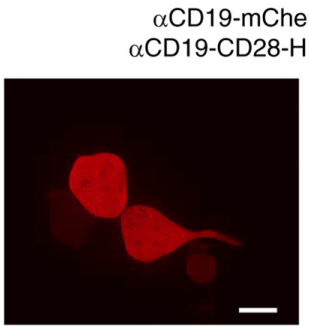

mCherry

$\mathrm{D}$

$\mathrm{F}$

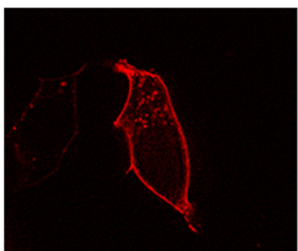

mCherry
B

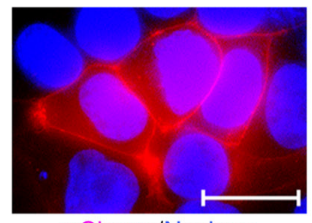

mCherry/Nucleus

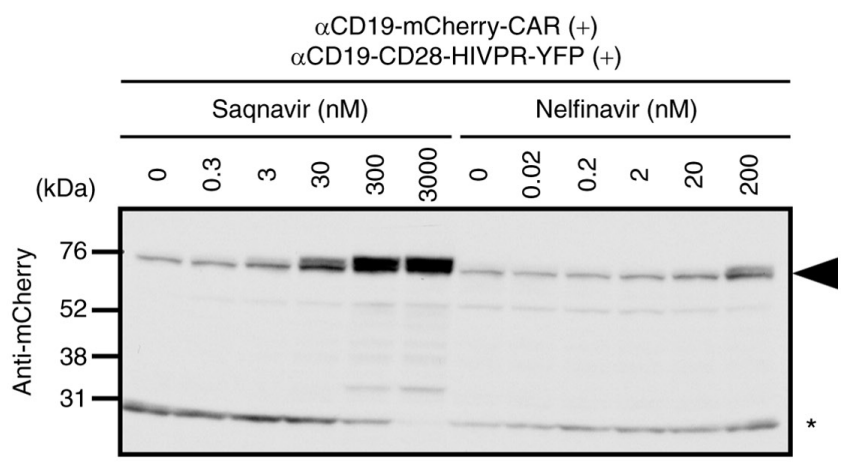

$\alpha \mathrm{CD} 19-\mathrm{mCherry}-\mathrm{CAR}(+)$ $\alpha$ CD19-Scissors-CAR-YFP $(+)$

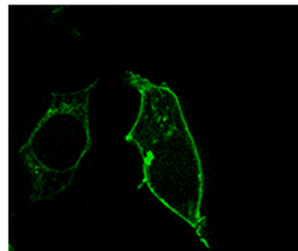

YFP

Figure 3. Cleavage of anti-CD19-mCherry-CAR by the HIVPR protease induces translocation of mCherry from the membrane to the cytoplasm. (A) A structure of anti-CD19-mCherry-CAR. The construct has a leader peptide (LP) sequence (white box), an anti-CD19 scFv (FMC63) domain (blue box), a CD28 transmembrane (TM) and inner-cellular (ICD) domains (orange box), the HIVPR recognition peptide sequence (green line) and a mCherry protein (pink box). (B) Localization of anti-CD19-mCherry-CAR. The anti-CD19-mCherry-CAR was expressed in 293T cells. Twenty-four hours after gene transduction, localizations of the mCherry-CAR (red color) were examined. Nuclei were stained with Hoechst 33258 (blue color). Scale bar, $20 \mu \mathrm{m}$. (C) Western blot analysis of fractionated cell lysates of $293 \mathrm{~T}$ cells expressing the indicated CAR constructs. Anti-CD19-CD28-HIVPR-YFP (Fig. 1) was used to express cytoplasmic HIVPR protease (indicated as HIVPR). Twenty-four hours after gene transduction, the cells were lysed. Plasma membrane fractions and cytosolic proteins were separated, and analyzed. Syntaxin 4 protein was used as an internal control of plasma membrane fractions. $\beta$-actin was used as an internal control of cytosolic proteins. An arrowhead on the right indicates the position of the full-length anti-CD19-mCherry-CAR (76 kDa); an asterisk on the right indicates the position of the cleaved mCherry (28 kDa). (D) Western blot analysis of cells treated with HIVPR inhibitors. Both anti-CD19-mCherry-CAR and anti-CD19-CD28-HIVPR-YFP were transfected into 293T cells. Twenty-four hours after gene transduction, the cells were treated with a HIVPR protease inhibitor, saquinavir or nelfinavir, at indicated concentrations. Twenty-four hours after the treatment, CARs and mCherry proteins were detected with an anti-mCherry antibody. A black arrowhead indicates the intact anti-CD19-mCherry-CARs (76 kDa). A black asterisk indicates the cleaved mCherry proteins (28 kDa). (E) Fluorescence microscopic examination of 293T cells with both anti-CD19-mCherry-CAR and anti-CD19-CD28-HIVPR-YFP. (F) Fluorescence microscopic examination of 293T cells with anti-CD19-mCherry-CAR and anti-CD19-Scissors-CAR-YFP. (E and F) Twenty-four hours after gene transduction of the indicated constructs, localizations of the mCherry (red color) and YFP (green color) were examined. Scale bar, $20 \mu \mathrm{m}$. TCL, total cell lysate; CE, cytosolic extract; PM, plasma membrane fraction CAR, chimeric antigen receptor.

expressing the Signal-CAR showed the YFP signal in the cytoplasm. To assess activity of the Signal-CAR, Jurkat cells stably expressing anti-CD19-Signal-CAR were established (Fig. S3A) and activation of the cells after co-cultivation with target cells was analyzed by measuring levels of CD69, a T-cell activation marker, on the cells (Fig. 5B and C). The Jurkat cells were not activated by co-cultivation with $\mathrm{K} 562$ cells, which lacked CD19 (Fig. 5C, left). By contrast, the Jurkat cells were activated by co-cultivation with the parental Raji cells expressing CD19 since levels of CD69 on the Jurkat cells were enhanced (Fig. 5C, right panels). Furthermore, to assess the effects of
Scissors-CAR, anti-HER2-Scissors-CAR was transduced into the Jurkat cells stably expressing anti-CD19-Signal-CAR. The Scissors-CAR, in which mCherry fluorescence protein was connected via the T2A sequence as a marker, was constructed (Fig. 5A, lower). Jurkat cells expressing both the CARs (Fig. S3B) were co-cultivated with target cells (Fig. 5D). When the Jurkat cells were co-cultivated with the parental Raji cells expressing CD19 alone, expression of CD69 on the CAR-Jurkat cells was not affected by the expression of Scissors-CAR (Fig. 5E, left). By contrast, when co-cultivated with the engineered Raji cells expressing both CD19 and 

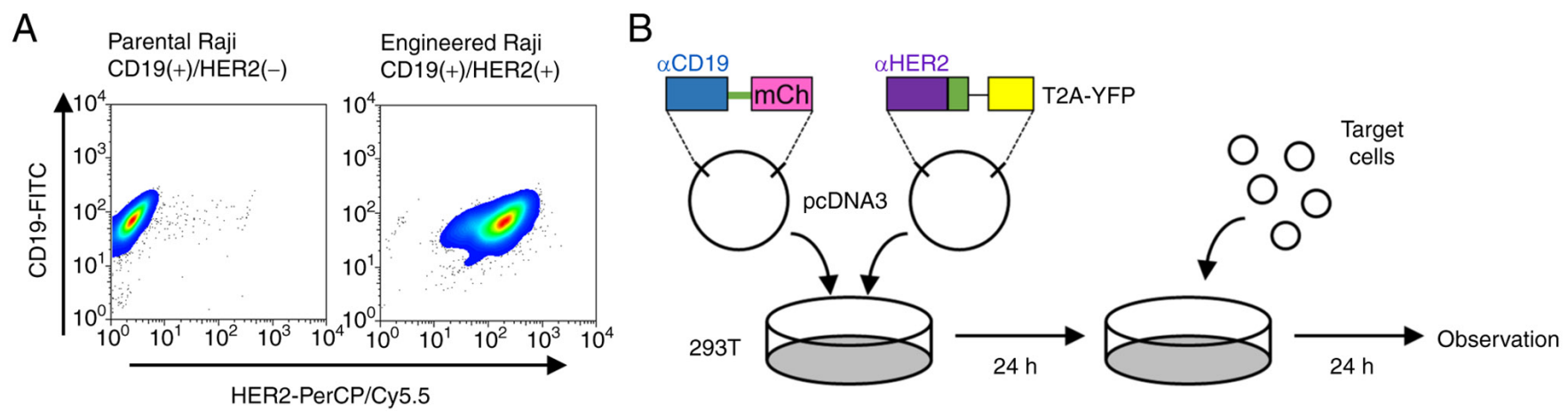

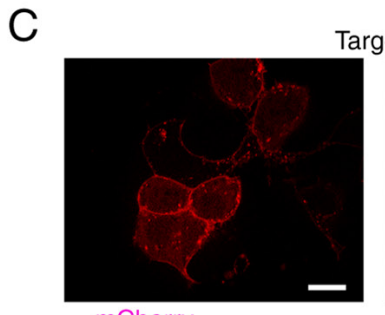

mCherry

Target cells: Raji CD19(+)/HER2(-)

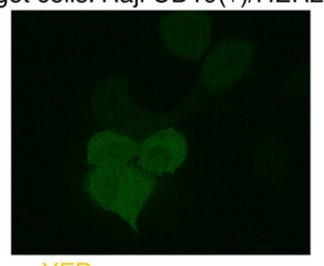

YFP

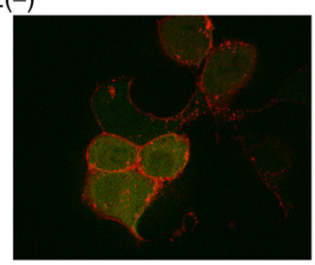

Merged

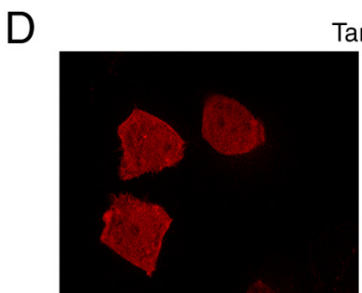

mCherry

Target cells: Raji CD19(+)/HER2(+)

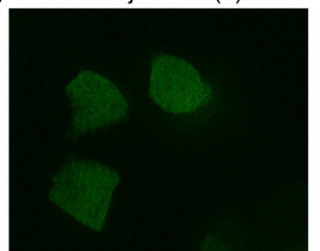

YFP

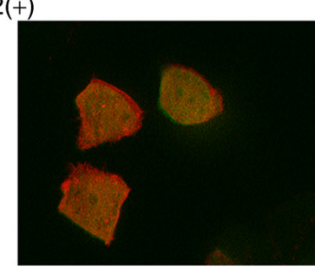

Merged

Figure 4. Anti-HER2-Scissors-CAR cleaves anti-CD19-mCherry-CAR when the cells are co-cultivated with target cells expressing both CD19 and HER2. (A) Flow cytometric analysis of CD19 (x-axis) and HER2 (y-axis) expression on target cells. pcDNA3(-)-HER2-Hygro vector was transduced into Raji cells to establish the engineered Raji cells (right panel). Results of the parental Raji cells are shown in the left panel. (B) A schema of the co-cultivation assays examining translocation of mCherry. Both anti-CD19-mCherry-CAR (left construct) and anti-HER2(4D5-3)-Scissors-CAR-T2A-YFP (right construct) were transduced into $293 \mathrm{~T}$ cells. Twenty-four hours after gene transduction, target cells were added into the culture dishes. Twenty-four hours after the co-cultivation, localization of mCherry was examined. A blue box: $\mathrm{scFv}$ domain against CD19, a green line: HIVPR recognition peptide sequence, a pink box: mCherry, a purple box: scFv domain against HER2, a green box: CD28( $\triangle \mathrm{ICD})$ and HIVPR $(\Delta 4)$ domains, a black horizontal line and a yellow box: T2A peptide and YFP. (C) The cells were co-cultivated with Raji cells expressing only CD19. (D) The cells were co-cultivated with the engineered Raji cells expressing both CD19 and HER2. (C and D) Red colors indicate mCherry in the left panels; green colors indicate YFP in the middle panels. Merged images are shown in the right panels. Scale bar, $20 \mu \mathrm{m}$. CAR, chimeric antigen receptor.

HER2, levels of CD69 on the CAR-Jurkat cells were significantly diminished by expression of Scissors-CAR (Fig. 5E, right; $\mathrm{P}=0.019$, Student's t-test, and Fig. S3C). Furthermore, expression of CD69 on the Jurkat cells expressing both the CARs was diminished not only by the engineered Raji cells (suspension cells) expressing HER 2 but also by the engineered SK-BR-3 cells (adhesion cells) expressing both the target proteins (Fig. S3D and E).

Scissors-CAR attenuates the cytotoxicity of primary CAR-T cells when the cells are co-cultivated with target cells expressing two distincT cell-surface proteins. To assess the cytotoxicity of CAR-T cells with Signal- and Scissors-CARs, the CARs were transduced into primary $\mathrm{T}$ cells, which were collected from healthy donors, using lentivirus. The manipulated $\mathrm{T}$ cells were co-cultivated with target cells (Fig. 6A). Cytotoxicity of the CAR-T cells against each the targeT cell was evaluated by measuring levels of lactate dehydrogenase (LDH) released from the target cells (Fig. 6B). The CAR-T cells with anti-CD19-Signal-CAR showed cytotoxicity against Raji cells, which showed statistical significance, but not K562 cells (Fig. S4A and Table SI). We constructed two types of Scissors-CARs using anti-HER2 (4D5-8) scFv with a high affinity to HER2 or anti-HER2 (4D5-3) scFv with a low affinity to HER2 (19). Lentiviruses encoding anti-CD19-Signal-CAR, in which YFP was connected via T2A sequence, and/or anti-HER2-Scissors-CAR, in which mCherry was connected via $\mathrm{T} 2 \mathrm{~A}$ sequence, were infected into primary $\mathrm{T}$ cells (Fig. S4B). CAR-T cells with both the CARs were sorted and co-cultivated with target cells. The CAR-T cells with anti-HER2-Scissors-CAR showed comparable cytotoxicity against the parental Raji cells only expressing CD19 to CAR-T cells without the Scissors-CAR (Fig. 6B, left). The CAR-T cells with the high affinity anti-HER2(4D5-8)-Scissors-CAR showed significant attenuation of cytotoxicity against the engineered Raji cells expressing both CD19 and HER2 (Fig. 6B, middle; $53.0 \pm 0.20 \%$ in 2:1 mixture, $\mathrm{P}=0.039$, one-way ANOVA with Dunnett's post-hoc test, control group=anti-CD19-Signal-CAR without Scissors-CAR). Furthermore, they showed attenuated cytotoxicity against the 
A $\alpha$ CD19-Signal-CAR-T2A-YFP

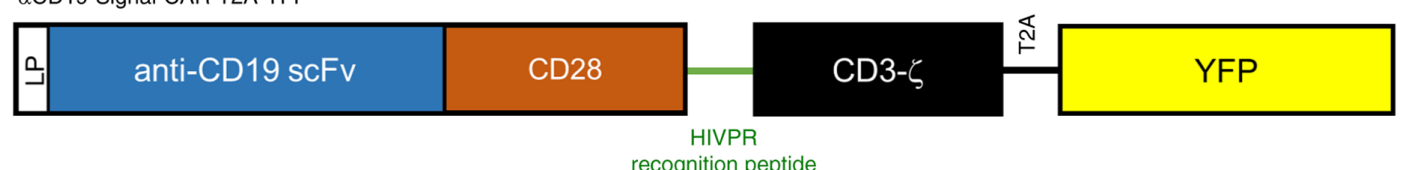

$\alpha$ HER2-Scissors-CAR-T2A-mCherry

\begin{tabular}{|c|c|c|c|}
\hline anti-HER2 scFv & $\mathrm{CD} 28(\Delta \mathrm{ICD})$ & $\operatorname{HIVPR}(\Delta 4)$ & mCherry \\
\hline
\end{tabular}

B

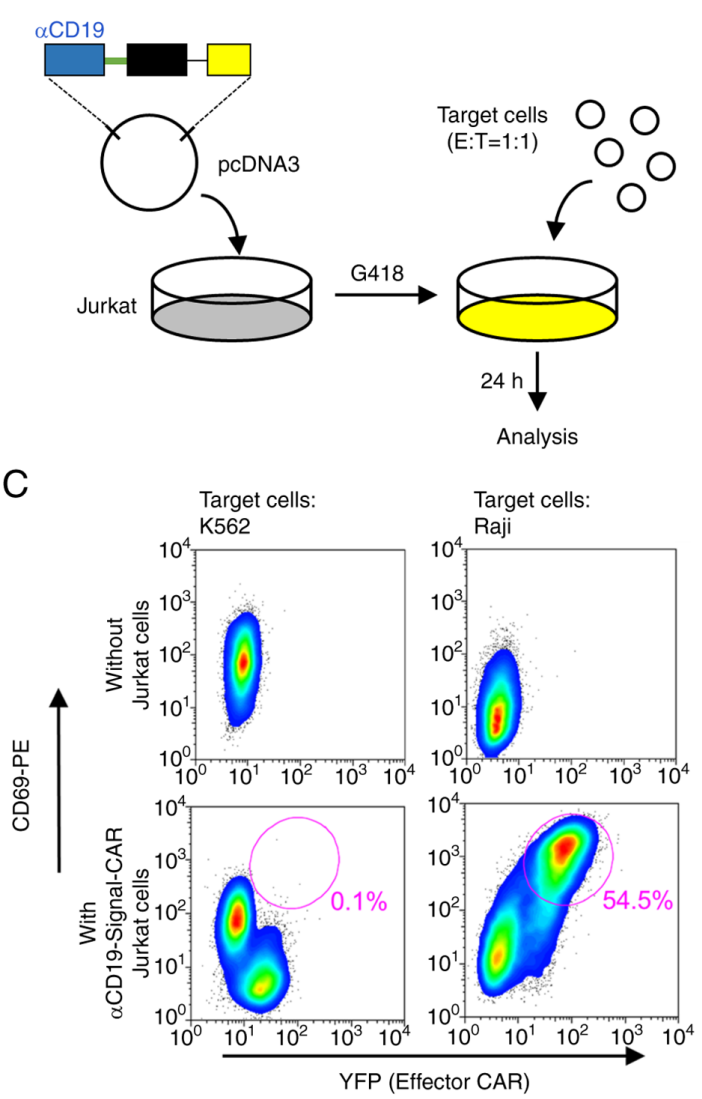

D

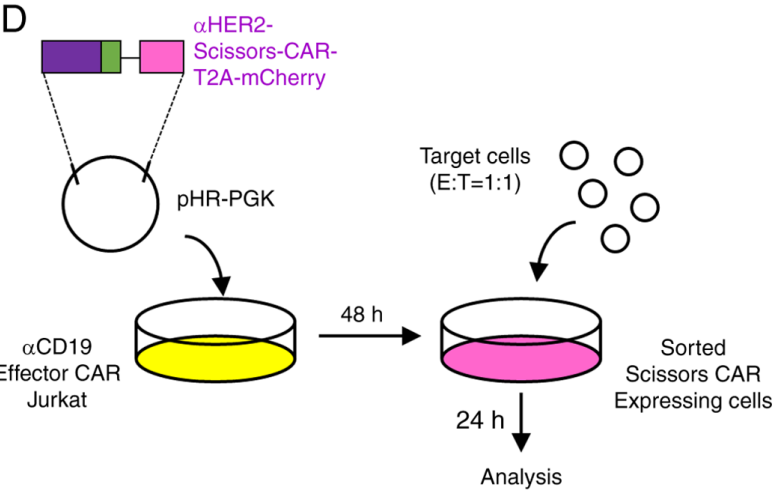

E

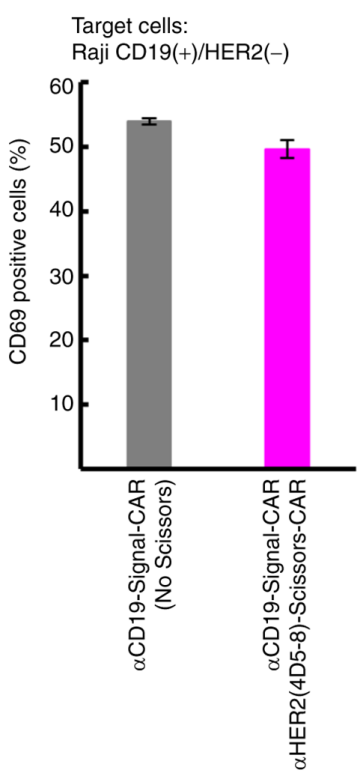

Target cells:

Raji CD19(+)/HER2(+)

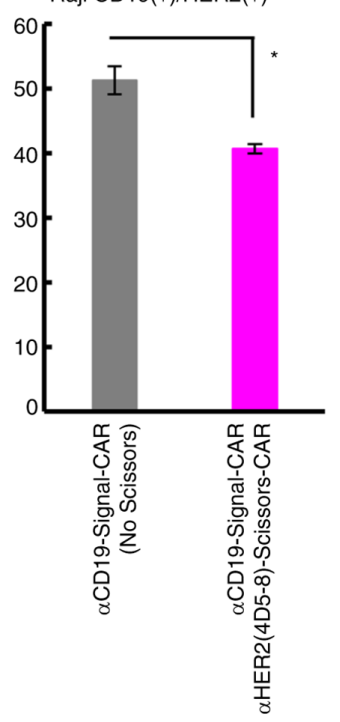

Figure 5. Anti-HER2-Scissors-CAR attenuates activation of Jurkat cells driven by anti-CD19-Signal-CAR when the cells are co-cultivated with target cells expressing both CD19 and HER2. (A) Structures of anti-CD19-Signal-CAR-T2A-YFP (upper) and anti-HER2-Scissors-CAR-T2A-mCherry (lower). These constructs have a leader peptide (LP) sequence, an anti-CD19 scFv (FMC63) or anti-HER2 scFv, a CD28 domain, a HIVPR recognition peptide sequence, and a CD3- $\zeta$ domain or HIVPR. YFP or mCherry protein was connected with the T2A sequence. Blue box, scFv domain against CD19; orange boxes, CD28 trans-membrane and co-stimulatory domains (upper) and CD28 trans-membrane domain lacking ICD (lower); a black box, CD3- $\zeta$ domain; a yellow box, YFP, a pink box, mCherry. A green horizontal line indicates the HIVPR recognition peptide sequence. Black horizontal lines indicate the T2A sequences. (B) A schema of co-cultivation assays using Jurkat cells stably expressing anti-CD19-Signal-CAR with target cells. The anti-CD19-Signal-CAR-T2A-YFP vector was transduced into Jurkat cells. The cells stably expressing the CAR were co-cultivated with target cells. Blue box, scFv domain against CD19; black box, CD3- $\zeta$ domain; yellow box, YFP. CD3- $\zeta$ and YFP are connected with the T2A sequence. Gray in the dish, culture medium containing parental Jurkat cells; yellow in the dish, culture media containing Jurkat cells expressing anti-CD19-Signal-CAR-T2A-YFP. (C) Activation of Jurkat cells with anti-CD19-Signal-CAR was examined by flow cytometry. Expression of YFP (x-axis) and CD69 (y-axis) was examined. Upper two panels show the data of target cells alone. Lower two panels show the data of Jurkat cells expressing anti-CD19-Signal-CAR that were co-cultivated with K562 cells (left panel) and Raji cells (right panel). Red circles indicate CD69-positive cells (percentages of positive cells are shown under the circles). (D) A schema of co-cultivation assays using Jurkat cells stably expressing both anti-CD19-Signal-CAR and anti-HER2-Scissors-CAR. Anti-HER2-Scissors-CAR-T2A-mCherry was introduced into Jurkat cells stably expressing anti-CD19-Signal-CAR-T2A-YFP. Forty-eight hours after infection, Jurkat cells expressing both YFP and mCherry were sorted and co-cultivated with target cells. Twenty-four hours after the co-cultivation, flowcytometric analysis was performed. Purple box, scFv domain against HER2; green box, $\mathrm{CD} 28(\Delta \mathrm{ICD})$ and HIVPR $(\Delta 4)$ domains; pink box, mCherry. HIVPR $(\Delta 4)$ domain and mCherry are connected with the T2A sequences. Yellow in the dish, culture medium containing Jurkat cells expressing anti-CD19-Signal-CAR-T2A-YFP; pink in the dish, culture media containing Jurkat cells expressing both anti-CD19-Signal-CAR-T2A-YFP and anti-HER2-Scissros-CAR-T2A-mCherry. (E) Levels of CD69 expression on Jurkat cells. Percentages of CD69-positive Jurkat cells are plotted. A left panel indicates results of co-cultivation with Raji cells expressing only CD19. A right panel indicates results of co-cultivation with the engineered Raji cells expressing both CD19 and HER2. Gray bars indicate Jurkat cells with only anti-CD19-Signal-CAR. Pink bars indicate Jurkat cells with both the Signal- and Scissors-CARs. Mean values \pm SD of three independent experiments are plotted. Data were analyzed using the Student's t-test and values showing significant difference are indicated as " $\mathrm{P}<0.05$. CAR, chimeric antigen receptor. 

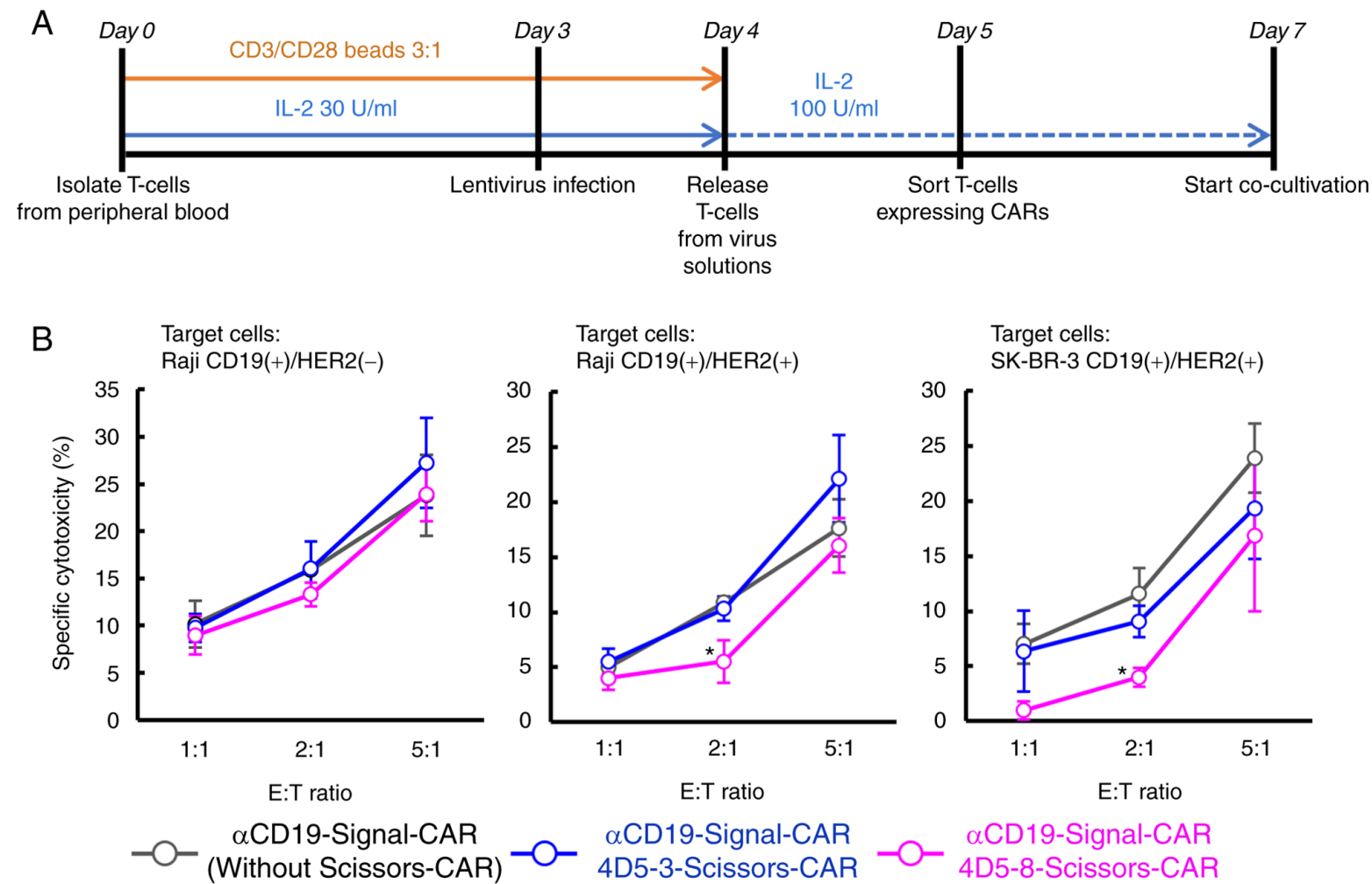

Figure 6. Cytotoxicity of primary CAR-T cells expressing both anti-CD19-Signal- and anti-HER2-Scissors-CARs is diminished by target cells expressing both CD19 and HER2. (A) A schema of the establishment of primary human CAR-T cells expressing both anti-CD19-Signal- and anti-HER2-Scissors-CARs, and co-cultivation assays to assess cytotoxicity of the CAR-T cells. To transduce two types of CARs, the same amounts of virus solutions of Signal- and Scissors-CARs were mixed and used for infection. Two days after infection, the cells expressing both the CARs were sorted. Four days after infection, the CAR-T cells were co-cultivated with target cells. Twenty to twenty-four hours after the co-cultivation, cytotoxicity against the target cells was analyzed. Primary T cells were treated with anti-CD3/CD28 beads (an orange arrow) and interleukin (IL)-2 (a blue arrow) for 4 days at indicated concentrations. A blue broken arrow indicates a period of treatment with IL-2 at an indicated concentration. (B) Target-cell-specific cytotoxicity of primary CAR-T cells. Specific cytotoxicity of the CAR-T cells (\%) are plotted. Values on the x-axis indicate ratio of Effector (E) and Target (T) cells. Results of co-cultivation with Raji cells expressing only CD19 (left panel), results of co-cultivation with the engineered Raji cells expressing both CD19 and HER2 (middle panel), and results of co-cultivation with the engineered SK-BR-3 cells expressing both CD19 and HER2 (right panel) are shown. Two types of scFv against HER2, 4D5-3 (blue lines) and 4D5-8 (pink lines) were used in Scissors-CARs. Results of anti-CD19 Signal-CAR-T cells without Scissors-CAR are shown with gray lines in each panel. Mean values \pm SD of three independent experiments are plotted. Data were analyzed using one-way ANOVA with Dunnett's post-hoc test for multiple comparison (control group=anti-CD19-Signal-CAR without Scissors-CAR) and values showing significant difference are indicated as ${ }^{*} \mathrm{P}<0.05$. CAR, chimeric antigen receptor.

engineered SK-BR-3 cells expressing both CD19 and HER2 as well (Fig. 6B, right; $43.2 \pm 0.17 \%$ in 2:1 mixture, $\mathrm{P}=0.039$, one-way ANOVA with Dunnett's post-hoc test, control group=anti-CD19-Signal-CAR without Scissors-CAR).

\section{Discussion}

High target-cell-specificity of chimeric antigen receptor (CAR)-T cells may improve the safety of CAR-T cell therapy, leading to expansion of treatable diseases by this immunotherapy. Our novel CAR-T cell system showed different cytotoxicity toward target cells depending on expression patterns of proteins on the target cells.

Previously, CAR systems recognizing multi-antigens have been reported including i) complement CARs, in which a cytoplasmic domain of CAR was divided into two components on two distinct CARs (20), ii) two-step CARs, in which the first CAR of a synthesized Notch receptor recognizes the first antigen, inducing expression of the second CAR recognizing the second antigen (21) and iii) inhibitory CARs with immuno-checkpoint proteins, PD-1 or CTLA-4, controlling activation of CAR-T cells (22). In the present study, we established the fourth strategy of CARs recognizing two distincT cell-surface proteins on target cells using a protease domain of HIVPR.

HIVPR is an exogenous protease derived from HIV that has been fully characterized. This enzyme has high specificity for the recognition peptide sequence. In addition, this enzyme becomes active only when it forms a homo-dimer $(15,23)$, which probably kept Scissors-CAR on the membrane inactive without contacting target cells. By contrast, HIVPR in the cytoplasm was probably automatically dimerized and cleaved the recognition peptide sequence.

In the present study, as a proof of principle, we used engineered cells expressing both CD19 and HER2 for our experiments. We successfully demonstrated that T-cell activation was controlled by patterns of protein-expression on target cells in our novel CAR-T cell system. Our system allows cells expressing two target proteins to escape from attacks by the CAR-T cells while tumor cells expressing only one protein fail to escape. In the treatment of B-cell malignancies, small fractions of normal B cells escaping from attacks by 
CAR-T cells would produce sufficient number of immunoglobulin. Therefore, partial attenuation of T-cell activation by our system would improve the safety of CAR-T cell therapies for B-cell malignancies.

We need to confirm that our system allows CAR-T cells to distinguish tumor cells from normal cells in vivo using immunocompromised animals before initiation of clinical trials. However, our current in vitro data suggest that it is too early to perform in vivo experiments since this study still has limitations to be overcome.

One is to define two distinct surface molecules expressed on normal cells while primary tumor cells only express one of the two proteins to apply this method to clinical practice. Since the herpes virus entry mediator (HVEM) protein has been reported to be expressed on normal B cells, but not on B-cell malignancies (24), this protein is a good candidate as a target protein for Scissors-CAR in B-cell malignancies. Since CD19 is expressed on both normal and neoplastic B cells, CD19 and HVEM could be good candidate surface molecules for development of this novel CAR-T therapy targeting B-cell malignancies.

The second is improvement of the suppressive effect of Scissors-CAR. Our study demonstrated that a higher amount of Scissors-CAR led to more efficient cleavage of Signal-CAR. However, the high levels of Scissors-CAR caused ligand-independent cleavage of Signal-CAR. Higher expression of Scissors-CAR may not be an optimal way to improve the suppressive effect. Therefore, improvement of binding affinity between Scissors-CAR and its ligand may increase the suppressive effect of Scissors-CAR.

Another way to increase the suppressive effect could be enhancement of proteolytic activity of HIVPR or the HIVPR recognition poly-peptide sequence used in this system. Amino-acid replacements in the protease domain and/or the recognition sequence may increase cleavage efficiency, leading to more potent suppression of Signal-CAR activity. Nevertheless, more modifications would be needed to improve the quality of our system.

Since the present study aimed to develop a novel system regulating CAR-signal based on the expression patterns of surface proteins on tumor/normal cells, we focused on the functional analysis of T cells such as CD69 expression or cytotoxicity. Therefore, we have not fully optimized/characterized several elements, including $\mathrm{scFv}$ binding affinity, cleavage efficiency and kinetics of the protease and its recognition sequence. Since our mCherry-CAR system is a beneficial tool with which to evaluate Scissors-CAR activity, more detailed analysis using this system could improve the quality of this novel system. Because of the COVID-19 pandemic, our research was restricted and several experiments we designed were not allowed to be performed in our institute. We hope that we will be able to fully optimize/characterize this system after this pandemic.

Currently, only several types of malignancies are effectively treated by CAR-T cell therapy. A number of clinicians and researchers are searching good targeT cell-surface proteins for CAR-T cell therapy. However, such tumor-specific cell-surface proteins are very rare and CAR-T cells unexpectedly attack important normal cells, causing severe adverse events in clinical trials $(25,26)$. Our system could help such clinicians and researchers to define good candidate target proteins for CAR-T cell therapies since only the proteins differentially expressed between tumor and normal cells need to be identified. Therefore, our system may expand the number of treatable diseases by CAR-T cell therapies.

In summary, our novel CAR-T cell system using a protease and the protease recognition peptide sequence allowed CAR-T cells to become active depending on the expression patterns of cell-surface proteins on target cells in vitro. This is one of the first steps to improve target-cell-specificity of this immunotherapy.

\section{Acknowledgements}

We thank Ms. Okada and Ms. Liu Meiou for the technical assistance. We also thank Mr. Sasaki and Mr. Ishitani for assistance with the cell sorting.

\section{Funding}

This research was supported by Nagao-Takeshi Research Grant (to YU), TMDU Young Investigator Research Grant (to YU), Takeda Research Grant (to KO), and Bristol-Myers-Squibb Research Grant (to NK).

\section{Availability of data and materials}

The datasets used and/or analyzed during the current study are available from the corresponding author on reasonable request.

\section{Authors' contributions}

SA, SY, DW, YU, KO, AN, OM and NK designed the experiments. SA and HL performed the experiments. SA and NK confirmed the authenticity of all the raw data and wrote the paper. All authors read and approved the final manuscript.

\section{Ethics approval and consent to participate}

These experiments were reviewed and approved by the Tokyo Medical and Dental University (TMDU) Ethics Committee (M2019-294).

\section{Patient consent for publication}

Not applicable.

\section{Competing interest}

The authors declare that they have no competing interests.

\section{References}

1. Maude SL, Laetsch TW, Buechner J, Rives S, Boyer M, Bittencourt H, Bader P, Verneris MR, Stefanski HE, Myers GD, et al: Tisagenlecleucel in children and young adults with B-Cell lymphoblastic leukemia. N Engl J Med 378: 439-448, 2018.

2. Park JH, Rivière I, Gonen M, Wang X, Sénéchal B, Curran KJ, Sauter C, Wang Y, Santomasso B, Mead E, et al: Long-term follow-up of CD19 CAR therapy in acute lymphoblastic leukemia. N Engl J Med 378: 449-459, 2018. 
3. Cappell KM, Sherry RM, Yang JC, Goff SL, Vanasse DA, McIntyre L, Rosenberg SA and Kochenderfer JN: Long-term follow-up of Anti-CD19 chimeric antigen receptor T-Cell therapy. J Clin Oncol 38: 3805-3815, 2020.

4. Kochenderfer JN, Dudley ME, Kassim SH, Somerville RP, Carpenter RO, Stetler-Stevenson M, Yang JC, Phan GQ, Hughes MS, Sherry RM, et al: Chemotherapy-refractory diffuse large B-cell lymphoma and indolent B-cell malignancies can be effectively treated with autologous T cells expressing an anti-CD19 chimeric antigen receptor. J Clin Oncol 33: 540-549, 2015.

5. Raje N, Berdeja J, Lin Y, Siegel D, Jagannath S, Madduri D, Liedtke M, Rosenblatt J, Maus MV, Turka A, et al: Anti-BCMA CAR T-Cell therapy bb2121 in relapsed or refractory multiple myeloma. N Engl J Med 380: 1726-1737, 2019.

6. Sterner RC and Sterner RM: CAR-T cell therapy: Current limitations and potential strategies. Blood Cancer J 11: 69, 2021.

7. Kochenderfer JN, Dudley ME, Feldman SA, Wilson WH, Spaner DE, Maric I, Stetler-Stevenson M, Phan GQ, Hughes MS Sherry RM, et al: B-cell depletion and remissions of malignancy along with cytokine-associated toxicity in a clinical trial of anti-CD19 chimeric-antigen-receptor-transduced T cells. Blood 119: 2709-2720, 2012.

8. Long AH, Haso WM, Shern JF, Wanhainen KM, Murgai M, Ingaramo M, Smith JP, Walker AJ, Kohler ME, Venkateshwara VR, et al: 4-1BB costimulation ameliorates $\mathrm{T}$ cell exhaustion induced by tonic signaling of chimeric antigen receptors. Nat Med 21: 581-590, 2015.

9. Lynn RC, Weber EW, Sotillo E, Gennert D, Xu P, Good Z, Anbunathan H, Lattin J, Jones R, Tieu V, et al: c-Jun overexpression in CAR T cells induces exhaustion resistance. Nature 576: 293-300, 2019.

10. Roybal KT, Williams JZ, Morsut L, Rupp LJ, Kolinko I, Choe JH, Walker WJ, McNally KA and Lim WA: Engineering T cells with customized therapeutic response programs using synthetic notch receptors. Cell 167: 419-432.e416, 2016.

11. Taube R, Zhu Q, Xu C, Diaz-Griffero F, Sui J, Kamau E, Dwyer M, Aird D and Marasco WA: Lentivirus display: Stable expression of human antibodies on the surface of human cells and virus particles. PLoS One 3: e3181, 2008.

12. Lindsten K, Uhlíková T, Konvalinka J, Masucci MG and Dantuma NP: Cell-based fluorescence assay for human immunodeficiency virus type 1 protease activity. Antimicrob Agents Chemother 45: 2616-2622, 2001.

13. Weissman AM, Hou D, Orloff DG, Modi WS, Seuanez H, O'Brien SJ and Klausner RD: Molecular cloning and chromosomal localization of the human T-cell receptor zeta chain: Distinction from the molecular CD3 complex. Proc Natl Acad Sci USA 85: 9709-9713, 1988

14. Conchello JA and Lichtman JW: Optical sectioning microscopy. Nat Methods 2: 920-931, 2005
15. Kanda Y: Investigation of the freely available easy-to-use software 'EZR' for medical statistics. Bone Marrow Transplant 48: 452-458, 2013

16. Navia MA and McKeever BM: A role for the aspartyl protease from the human immunodeficiency virus type 1 (HIV-1) in the orchestration of virus assembly. Ann NY Acad Sci 616: 73-85, 1990.

17. Wilkins MR, Gasteiger E, Bairoch A, Sanchez JC, Williams KL, Appel RD and Hochstrasser DF: Protein identification and analysis tools in the ExPASy server. Methods Mol Biol 112: 531-552, 1999.

18. Liu Z, Chen O, Wall JB, Zheng M, Zhou Y, Wang L, Vaseghi HR, Qian L and Liu J: Systematic comparison of 2A peptides for cloning multi-genes in a polycistronic vector. Sci Rep 7: 2193 , 2017.

19. Carter P, Presta L, Gorman CM, Ridgway JB, Henner D, Wong WL, Rowland AM, Kotts C, Carver ME and Shepard HM: Humanization of an anti-p185HER2 antibody for human cancer therapy. Proc Natl Acad Sci USA 89: 4285-4289, 1992.

20. Cho JH, Collins JJ and Wong WW: Universal chimeric antigen receptors for multiplexed and logical control of $\mathrm{T}$ cell responses. Cell 173: 1426-1438.e1411, 2018.

21. Choe JH, Watchmaker PB, Simic MS, Gilbert RD, Li AW, Krasnow NA, Downey KM, Yu W, Carrera DA, Celli A, et al: SynNotch-CAR T cells overcome challenges of specificity, heterogeneity, and persistence in treating glioblastoma. Sci Transl Med 13: eabe7378, 2021.

22. Fedorov VD, Themeli $M$ and Sadelain M: PD-1- and CTLA-4-based inhibitory chimeric antigen receptors (iCARs) divert off-target immunotherapy responses. Sci Transl Med 5: 215ra172, 2013.

23. Mager PP: The active site of HIV-1 protease. Med Res Rev 21: 348-353, 2001.

24. Boice M, Salloum D, Mourcin F, Sanghvi V, Amin R, Oricchio E, Jiang M, Mottok A, Denis-Lagache N, Ciriello G, et al: Loss of the HVEM tumor suppressor in lymphoma and restoration by modified CAR-T cells. Cell 167: 405-418.e413, 2016.

25. Lamers CH, Klaver Y, Gratama JW, Sleijfer S and Debets R: Treatment of metastatic renal cell carcinoma (mRCC) with CAIX CAR-engineered T-cells-a completed study overview. Biochem Soc Trans 44: 951-959, 2016.

26. Morgan RA, Yang JC, Kitano M, Dudley ME, Laurencot CM and Rosenberg SA: Case report of a serious adverse event following the administration of $T$ cells transduced with a chimeric antigen receptor recognizing ERBB2. Mol Ther 18: 843-851, 2010.

This work is licensed under a Creative Commons Attribution-NonCommercial-NoDerivatives 4.0 International (CC BY-NC-ND 4.0) License. 